\title{
Blood-based RAS mutation testing: concordance with tissue-based RAS testing and mutational changes on progression
}

\author{
Theodora Germetaki1 (iD), Camille Nicholls, Richard A Adams², Michael Braun, Jane \\ Rogan $^{1}$, Sharzad Moghadam¹, Eva Lenfert ${ }^{3}$, Antje Lukas $^{3}$, Daniel L Edelstein ${ }^{3}$, Frederick S \\ Jones $^{3} \&$ Mark P Saunders $*, 1$ \\ ${ }^{1}$ Department of Medical \& Clinical Oncology, The Christie Hospital, 550 Wilmslow Road, Manchester M20 4BX, UK \\ ${ }^{2}$ Department of Medical Oncology, Velindre Hospital, Velindre Road, Cardiff, Wales CF14 2TL, UK \\ ${ }^{3}$ Sysmex Inostics GmbH, Falkenried 88, Hamburg 20251, Germany \\ *Author for correspondence: Tel.: 0161446 3357; mark.saunders@christie.nhs.uk
}

\begin{abstract}
Aim: To determine the concordance between plasma and tissue RAS mutation status in metastatic colorectal cancer patients to gauge whether blood-based testing is a viable alternative. We also evaluated the change in mutation status on progression. Materials/methods: RAS testing was performed on plasma from patients commencing first-line therapy (OncoBEAM ${ }^{T M}$ RAS CEIVD kit). Results were then compared with formalin-fixed paraffin embedded tumor samples. Results: The overall percentage agreement (concordance) was $86.0 \%(86 / 100)$, which demonstrates that blood-based testing is an alternative to tissuebased testing. Reproducibility was $100 \%$ between three laboratories and $20 \%$ showed changes in their RAS mutational status on progression. Conclusion: These results show good concordance between tissue and plasma samples and suggest the need for longitudinal plasma testing during treatment to guide management decisions.
\end{abstract}

First draft submitted: 22 May 2020; Accepted for publication: 2 July 2020; Published online:

27 July 2020

Keywords: BEAMing $\bullet$ circulating-free DNA $\bullet$ colon cancer $\bullet \mathrm{ctDNA} \bullet \mathrm{mCRC} \bullet$ RAS

Randomized controlled trials have demonstrated the benefit of anti-EGFR monoclonal antibodies such as cetuximab and panitumumab as single agents or in combination with other chemotherapeutic regimens in metastatic colorectal cancer (mCRC) patients [1-3]. Prior to initiation of anti-EGFR therapy, RAS (KRAS, Kirsten RAt Sarcoma virus; $N R A S$, Neuroblastoma RAt Sarcoma virus) genotyping is performed and mutations are detected in approximately $50 \%$ of patients with mCRC $[4,5]$.

$R A S$ mutation detection in routine clinical practice is usually performed using DNA extracted from formalin-fixed paraffin embedded (FFPE) tumor specimens [6]. Tissue processing, however, presents several practical challenges, including poor tissue quality, extensive workflow, lack of standardization of testing methodologies and delays in treatment. Results from Europe revealed that $48 \%$ of laboratories failed to complete $R A S$ genotyping within 14 days [7]. From a biological perspective, accurate genotype determination can be obscured by tumor molecular heterogeneity [8]. A single tumor site may contain multiple clones with varying mutational status. A single tissue biopsy may therefore not represent true mutational burden, particularly in patients with tumor recurrence and multiple metastases [9]. Thus, for a variety of reasons, the aggregate of data suggests that although tissue has traditionally served as the primary material for $R A S$ testing, it has limitations. In patients with metastatic disease, an accurate blood-based $R A S$ assessment may overcome these limitations.

As tumor cells die, they release mutant tumor DNA fragments into the blood. Given that circulating tumor DNA (ctDNA) fragments are readily detected in patients with advanced cancers, detection of mutations in ctDNA provides an excellent biomarker to track patients with metastatic disease [10]. Recent studies also indicate that ctDNA testing provides rapid genotype results for mCRC patients that accurately reflect the mutation status of tumor tissue [11-14]. 
Among the various plasma ctDNA assays, the digital PCR-based BEAMing assay (beads, emulsions, amplification and magnetics) has been clinically validated across several tumor types including colorectal cancer (CRC). The OncoBEAM ${ }^{T M}$ RAS CRC plasma assay (Sysmex Inostics), based on the BEAMing technology [15], is a CE-marked IVD liquid biopsy test that provides a systemic evaluation of $R A S$ tumor mutation status from a blood sample in less than 7 days, enabling effective and timely evaluation of a patient's eligibility for anti-EGFR therapy.

The aim of the study was to demonstrate the utility of a standardized blood-based RAS genotyping test as an alternative to tissue-based $R A S$ genotyping prior to treatment with anti-EGFR therapy. We also cross-checked the concordance of results between three independent laboratories and evaluated the longitudinal variation of $R A S$ mutant allelic fraction (MAF) during progression to assess the prognostic value of serial plasma $R A S$ analyses.

\section{Materials \& methods}

Patient inclusion criteria

Approval was obtained from Manchester Cancer Research Biobank and eligible patients with untreated mCRC (could have had previous adjuvant chemotherapy) gave informed consent for blood to be drawn as part of this study.

\section{Plasma tumor sampling}

Approximately $30 \mathrm{ml}$ of blood was collected in three Streck tubes and the plasma was prepared according to OncoBEAM RAS CRC kit instructions. Independent studies have previously demonstrated the stability and shipping requirements of collected blood and Sysmex provided a detailed protocol for plasma preparation. Plasma was stored in $2 \mathrm{ml}$ aliquots at $-80^{\circ} \mathrm{C}$ until analysis. Additional blood samples were obtained in cases where patients exhibited radiological and/or clinical disease progression. Although only 2 to $3 \mathrm{ml}$ of plasma are required for $R A S$ mutation testing using the OncoBEAM RAS IVD, several residual and replicate samples were made available for further accuracy and reproducibility studies performed in this study.

\section{Tissue RAS mutation testing}

Tissue $R A S$ mutational analysis was performed as per standard of care using the QIAGEN Therascreen Pyro Kit, which detects mutations in exons $2 \& 3$ in KRAS and NRAS genes; a $\%$ mutant allele threshold was used as the cut-off for calling mutations. Additional testing to detect $R A S$ mutations in exon 4 was performed using tissue BEAMing; for this analysis, a $1 \%$ mutant allele threshold was used as the cut-off for calling mutations. The $1 \%$ cut-off for tissue BEAMing was demonstrated in the Phase III CRYSTAL and OPUS studies [16,17]. With respect to the clinical relevance of the $1 \%$ cut-off for BEAMing, in a retrospective analysis of the CRYSTAL trial, patients with tumor RAS MAFs between 0.1 and $<5 \%$ were more likely to benefit from the addition of cetuximab to FOLFIRI [16].

\section{Concordance of ctDNA in three laboratories}

Tumor-specific circulating DNA is distinguished from normal DNA by the occurrence of point mutations in tumor-derived DNA. The OncoBEAM RAS CRC IVD assay detects the MAF of ctDNA in a high background of normal DNA with high sensitivity and specificity. In this study, a panel of 34 frequent point mutations in the KRAS and NRAS genes are detected and used to identify and monitor the fraction of mutant DNA and normal DNA. Tumor-specific circulating DNA is measured by the BEAMing assay as a continuous variable and represented as a ratio between the mutant and wild-type allele evaluated. Plasma samples were analyzed for 34 mutations in KRAS and NRAS (codons 12, 13, 59, 61, 117 and 146) using the OncoBEAM RAS CRC CEIVD assay (Sysmex Inostics, Hamburg, Germany). A subset of plasma samples (replicates) were also tested independently at two other OncoBEAM testing centers, Genomic Diagnostics Laboratory (GDL, Manchester, UK) and IMP laboratory (Sysmex, Kobe, Japan), to examine the robustness of the quantitative values of the MAF obtained from $R A S$-mutation-positive CRC patients.

The OncoBEAM RAS CRC IVD kit is based on BEAMing, which is a flow cytometric emulsion digital PCR technique in which single molecule PCR reactions are performed on magnetic beads in water-in-oil emulsions [18]. BEAMing utilizes emulsion digital PCR performed on magnetic beads to amplify single DNA molecules. Individual beads are then hybridized to allele-specific fluorescently labeled probes complementary to the mutant and wild-type DNA sequences. Finally, the bead population is analyzed by flow cytometry to count and sort wild-type and mutant beads. 
Although it has been shown that BEAMing can detect one mutant molecule in a background of 10,000 wild-type molecules [15], the setting of cut-offs for each of the $34 R A S$ mutations analyzed in the assay were determined to be between 0.02 and $0.04 \%$ MAF; this ensures that the limits of detection (LODs) for each of the 34 RAS mutations are well above background signals or limits of blank (LoBs) for each analyte to be detected in clinical samples. LODs were determined by probit regression analyses by spiking wild-type (non- $R A S$ mutation-containing) plasma samples with each RAS analyte. Background signals (LoBs) were determined in DNA prepared from wild-type plasma samples lacking $R A S$ mutations at low, medium and high concentrations of genomic DNA. Based on the results of these experiments, cut-offs of $0.02-0.04 \%$ were observed to be appropriate so as to obtain a $95 \%$ probability/CI of reporting a "mutation detected" result (OncoBEAM RAS CRC kit instructions for use, Sysmex Inostics GmbH, Hamburg, Germany). Between 2 and $3 \mathrm{ml}$ of plasma from each sample were used for each assay.

$R A S$ mutation results are reported as both the absolute quantity of mutant beads and the fractional abundance of mutant DNA alleles relative to wild-type DNA alleles. The result is reported as the fractional abundance of mutant DNA alleles relative to wild-type DNA alleles in a plasma sample. To generate the ratio of mutant to wild-type DNA alleles (MAF), $\sim 3 \times 10^{6}$ beads are interrogated in each BEAMing analysis (corresponding to $\sim 90,000$ beads per mutation). The absolute number of RAS mutant alleles are not reported by BEAMing, as the determination of mutant status is dependent on the total amount DNA in an individual sample. Total circulating DNA levels (both wild-type and mutant) are subject to interpatient variability, which may be directly related to tumor burden or other characteristics such as inflammation and immune response.

\section{Statistical analysis of concordance}

Concordance of $R A S$ mutation status was determined by calculating the agreement of RAS mutant and wild-type cases between tissue and plasma specimens. For discordant cases, the mutational status of both tissue and blood samples was rechecked using BEAMing. The modified Wald method was used to determine boundaries for 95\% $\mathrm{CI}$ for each of the following three proportions: overall percentage agreement (OPA), positive percentage agreement (PPA) and negative percentage agreement (NPA). The MAF values for newly diagnosed versus recurrent mCRC patients were evaluated by calculating mean MAF values with standard errors and compared with p-values derived using a Welch unequal variances t-test. All statistical tests were two-sided; the threshold for statistical significance was $\mathrm{p}<0.05$.

\section{Results}

\section{Patients}

In total, 104 patients (58 males and 46 females) with a median age of 65 years and PS $0-1$ were entered into this study. However, only 100 patients were included in the concordance analysis; four cases were excluded because either the corresponding tissue samples were unavailable $(\mathrm{n}=3)$ or the baseline plasma $R A S$ result was not available ( $\mathrm{n}=1$; Table 1$)$.

49 patients had $R A S$ wild-type (RAS-WT) tumors and 51 had mutations of the RAS gene ( $m R A S$; Table 2). The median interval between obtaining a tissue sample and initiation of first-line treatment was 66.5 days. The median interval between obtaining a plasma sample and the start date of first-line treatment was 1 day (range: 0-104 days; mean: 4.13 days; Table 3).

\section{RAS mutation analyses}

The concordance of $R A S$ status between matched plasma and tissue samples from each patient is summarized in Table 4. The RAS mutation status determined by OncoBEAM plasma testing versus the reference standard method performed on FFPE tissue samples was concordant in 86 out of 100 cases (86\% OPA), with 86.2 PPA and 85.7\% NPA. 14 discordant $R A S$ mutation results were observed between plasma and tissue testing. Seven patients showed $m R A S$ status in tissue but were $R A S$-WT in plasma (plasma false negatives). Conversely, plasma testing revealed $m R A S$ status in seven patients whose tumors were determined $R A S$-WT by tissue testing (plasma false positives). For two out of three patients showing plasma false positives for which an additional plasma sample was available for testing at a later time point, the same $R A S$ mutations were also detected. This suggests that low-frequency $R A S$ mutations were likely to be present in the tumors of these patients below the limit of detection of the SOC tissue testing method. Recent data has suggested that low RAS frequency mutations may be a common occurrence in $\mathrm{mCRC}[19,20]$. 


\begin{tabular}{|c|c|c|}
\hline Variable & $\mathrm{n}$ & $\%$ \\
\hline Total number of patients & $104^{\dagger}$ & 100 \\
\hline Male & 58 & 55.8 \\
\hline Female & 46 & 44.2 \\
\hline Age (median, range) & $65(31$ & \\
\hline \multicolumn{3}{|l|}{ TNM staging at initial diagnosis: } \\
\hline$-T x$ & 23 & 22.1 \\
\hline$-\mathrm{T} 1$ & 1 & 1.0 \\
\hline$-\mathrm{T} 2$ & 4 & 3.8 \\
\hline$-\mathrm{T} 3$ & 40 & 38.5 \\
\hline$-\mathrm{T} 4$ & 36 & 34.6 \\
\hline$-N x$ & 24 & 23.1 \\
\hline- No & 14 & 13.5 \\
\hline$-N 1$ & 24 & 23.1 \\
\hline$-\mathrm{N} 2$ & 42 & 40.3 \\
\hline \multicolumn{3}{|l|}{ Metastatic lesions at trial entry: } \\
\hline - Presented with metastatic colorectal cancer & 70 & 67.3 \\
\hline - Relapsed after adjuvant treatment & 34 & 32.7 \\
\hline \multicolumn{3}{|l|}{ Performance status: } \\
\hline - PSO & 45 & 43.3 \\
\hline - PS1 & 45 & 43.3 \\
\hline - PS2 & 12 & 11.5 \\
\hline - Unknown PS & 2 & 1.9 \\
\hline \multicolumn{3}{|l|}{ First-line treatment: } \\
\hline$-\operatorname{IrMdG}$ & 20 & 19.2 \\
\hline - IrMdG + cetuximab & 15 & 14.4 \\
\hline - Capecitabine & 8 & 7.7 \\
\hline - OxCap & 5 & 4.8 \\
\hline - OxMdG & 33 & 31.7 \\
\hline - OxMdg + panitumumab & 9 & 8.7 \\
\hline $\begin{array}{l}\text { - Other treatments (IrMdG + masitinib, OxMdG + cetuximab, capecitabine + bevacizumab, irinotecan single agent, } \\
\text { IrMdG + AZD8931, bevacizumab + trifluridine + tipiracil, cetuximab single agent) }\end{array}$ & 13 & 12.5 \\
\hline - Unknown & 1 & 1.0 \\
\hline
\end{tabular}

\begin{tabular}{|c|c|c|}
\hline Tissue sample & Patients (n) & $\%$ \\
\hline Wild type & 52 & 50.0 \\
\hline Mutant & 52 & 50.0 \\
\hline Resection tissue & 39 & 37.5 \\
\hline Biopsy tissue & 61 & 58.7 \\
\hline Unknown tissue type & 4 & 3.8 \\
\hline Sample from primary tumor & 89 & 85.6 \\
\hline Sample from metastatic lesion & 12 & 11.5 \\
\hline Unknown & 3 & 2.9 \\
\hline
\end{tabular}

Plasma mutant allele fraction analyses in three laboratories

A useful feature of ctDNA analysis using OncoBEAM is the ability to determine the MAF of cell-free mutant alleles as a proportion of the overall cell-free DNA content in circulation at the time of sampling. To examine the reproducibility of plasma $R A S$ mutation results and to assure accuracy of $R A S$ MAF values, replicate plasma samples 
Table 3. Time from tissue/plasma to treatment.

\begin{tabular}{|l|l|}
\hline Intervals & Patients $(\mathbf{n})$ \\
\hline $1-4$ days (\%) & Median: 1 day (range: $0-104$ days; mean: 4.13 days) \\
\hline $5-10$ days (\%) & $72(69.2)$ \\
\hline$>10$ days $(\%)$ & $12(11.5)$ \\
\hline After treatment $(\%)$ & $12(11.5)$ \\
\hline NA $(\%)$ & $3(2.9)$ \\
\hline Between tissue and treatment & $5(4.8)$ \\
\hline & \\
\hline NA: Not applicable. & Median: 66.5 days (range: 4-1990 days) \\
\hline
\end{tabular}

\begin{tabular}{|c|c|c|c|c|c|c|}
\hline \multirow[t]{2}{*}{ RAS } & \multicolumn{2}{|c|}{ Plasma ctDNA RAS result } & \multirow[t]{2}{*}{ Total } & \multicolumn{3}{|c|}{ Tumor-tissue $R A S$ result } \\
\hline & Mutant & WT & & PPA $(95 \% \mathrm{Cl})$ & NPA $(95 \% \mathrm{Cl})$ & OPA $(95 \% \mathrm{Cl})$ \\
\hline Mutant & 44 & 7 & 51 & $100 \times 44 / 51$ & $100 \times 42 / 49$ & $100 \times 86 / 100$ \\
\hline WT & 7 & 42 & 49 & $86.2 \%$ & $85.7 \%$ & $86.0 \%$ \\
\hline Total & 51 & 49 & 100 & $(74 \%-94 \%)$ & $(73 \%-93 \%)$ & $(78 \%-92 \%)$ \\
\hline
\end{tabular}

Figure 1. Bar chart showing the average plasma DNA-mutant fractions detected in ctDNA of patients with newly diagnosed compared with recurrent disease and the overall cohort of patients with RAS mutations detected in the plasma by BEAMing. The average value and standard error (SE) for RAS MAF for all patients $(\mathrm{n}=48$; overall) in which RAS mutations were detected by OncoBEAM RAS CRC assay is represented by the lilac bar on the right (mean: $9.56 \pm 1.70 \mathrm{SE}$ ). The average values and SEs for RAS MAF from newly diagnosed $\mathrm{mCRC}$ patients with primary tumor intact (prior to

surgery; $n=26$ ) and patients with primary tumor removed (post-surgery) and or patients with recurrent disease/metastases only $(n=22)$ are represented by the violet bar on the left (mean: $15.12 \pm 2.61 \mathrm{SE}$ ) and the medium purple bar in the middle (mean MAF: $3.03 \pm 0.79 \mathrm{SE}$ ), respectively. $p=0.0001$ for MAF\% in newly diagnosed patients compared with those presenting with recurrent disease/metastases only. The $p$-values were derived from a Welch unequal variances t-test.

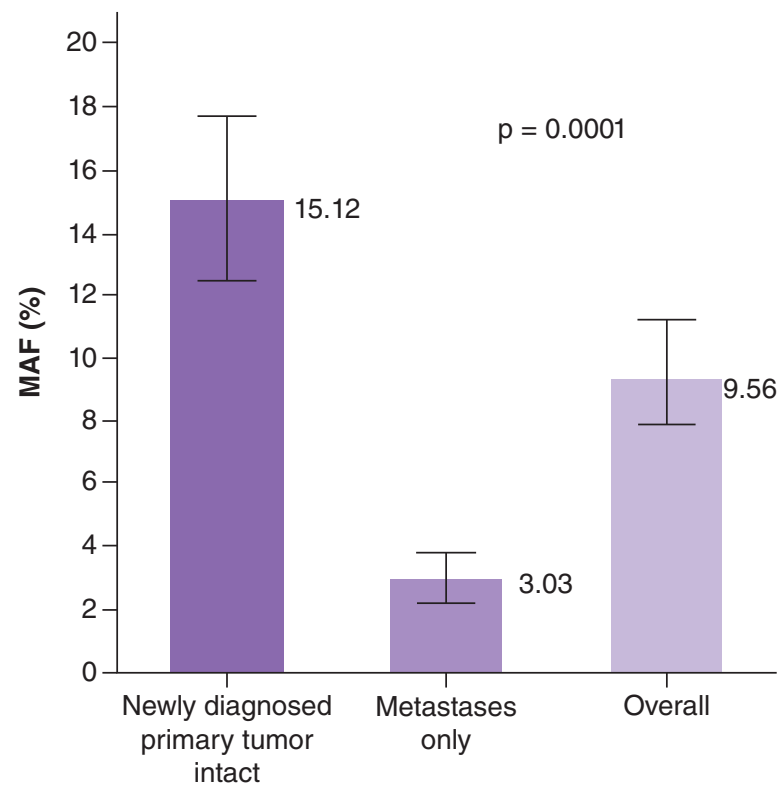

MAF: Mutant allelic fraction.

were evaluated and compared at three separate laboratories (UK, Germany, Japan). As shown in Table 5, results from testing of 76 replicate plasma samples from $27 \mathrm{mCRC}$ patients at the three different laboratories showed $100 \%$ agreement, with only one of the tests $(1.3 \%)$ giving an invalid result. To further evaluate the accuracy of MAF values, 38 replicates from $13 R A S$-mutation-positive patients were examined in the three separate laboratories. As shown in Table 5, the average variance in MAF values was no more than $+/-13.4 \%$ (95 CI; $9.1-17.8 \%$ ). This low variance was consistent for MAF values over three orders of magnitude of ctDNA concentration present in the samples.

For 51 patients with $R A S$ mutations, the average MAF value was $9.56 \%$ (Figure 1). Since the frequency of circulating mutant alleles might be related to overall tumor burden or extent of metastatic invasion, the mean MAF 
Table 5. Comparison of mutant allelic fraction percentages obtained in replicate sample testing using the OncoBEAM RAS CRC assay across three different laboratories.

\begin{tabular}{|c|c|c|c|c|c|c|c|c|}
\hline Patient ID & Tissue result & $\begin{array}{l}\text { GDL plasma } \\
\text { result }\end{array}$ & GDL MAF\% & $\begin{array}{l}\text { IMP plasma } \\
\text { result(s) }{ }^{\dagger}\end{array}$ & IMP MAF (\%) & SI plasma result & SI MAF (\%) & Mean MAF \\
\hline PT2163 & $R A S-W T$ & & & $R A S-W T$ & & $R A S-W T$ & & \\
\hline PT2654 & KR12+ & KR12+ & 42.435 & KR12+ & $48.011,45.194$ & KR12+ & 51.889 & 46.88 \\
\hline PT2679 & $R A S-W T$ & & & RAS-WT & & $R A S-W T$ & & \\
\hline PT2716 & KR12+ & & & KR12+ & $21.09,19.403$ & KR12+ & 27.62 & 22.7 \\
\hline PT2732 & $R A S-W T$ & & & $R A S-W T$ & & $R A S-W T$ & & \\
\hline PT2733 & KR12+ & KR12+ & 6.756 & KR12+ & $8.645,8.466$ & KR12+ & 11.69 & 8.89 \\
\hline PT2738 & $R A S-W T$ & & & $R A S-W T$ & & $R A S-W T$ & & \\
\hline РT2794 & KR12+ & KR12+ & 0.809 & KR12+ & 0.692 & KR12+ & 0.6549 & 0.7186 \\
\hline PT2814 & No result & KR12+ & 11.743 & KR12+ & 1.792 & KR12+ & 8.9684 & 7.5011 \\
\hline PT2815 & $R A S-W T$ & $R A S-W T$ & & $R A S-W T$ & & $R A S-W T$ & & \\
\hline PT2817 & $R A S-W T$ & & & $R A S-W T$ & & $R A S-W T$ & & \\
\hline PT2821 & $R A S-W T$ & $R A S-W T$ & & $R A S-W T$ & & $R A S-W T$ & & \\
\hline РT3269 & KR12+ & KR12+ & 9.564 & KR12+ & 10.65 & KR12+ & 8.771 & 9.662 \\
\hline РT3272 & $R A S-W T$ & $R A S-W T$ & & $R A S-W T$ & & $R A S-W T$ & & \\
\hline РТ3273 & No result & KR13+ & 14.674 & KR13+ & 15.589 & KR13+ & 16.036 & 15.433 \\
\hline РT3274 & $R A S-W T$ & RAS-WT & & $R A S-W T$ & & $R A S-W T$ & & \\
\hline РT3275 & KRASG13D & KR13+ & 0.232 & KR13 & 0.28 & KR13+ & 0.187 & 0.233 \\
\hline РТ3276 & $R A S-W T$ & & & $R A S-W T$ & & $R A S-W T$ & & \\
\hline РТ3277 & KRASG12D & KR12+ & 11.446 & KR12+ & $13.905,12.294$ & KR12+ & 14.092 & 12.923 \\
\hline РТ3278 & $R A S-W T$ & $R A S-W T$ & & $\begin{array}{l}R A S-W T \\
R A S-W T\end{array}$ & & $R A S-W T$ & & \\
\hline РT3279 & $R A S-W T$ & $R A S-W T$ & & $\begin{array}{l}R A S-W T \\
R A S-W T\end{array}$ & & $R A S-W T$ & & \\
\hline РT3280 & $R A S-W T$ & $R A S-W T$ & & $\begin{array}{l}\text { RAS-WT, } \\
\text { invalid }^{\ddagger}\end{array}$ & & $R A S-W T$ & & \\
\hline РT3281 & $R A S-W T$ & $R A S-W T$ & & & & RAS-WT & & \\
\hline РТ3282 & No result & KR12+ & 11.088 & & & KR12+ & 10.001 & 10.5445 \\
\hline РT3283 & KRASG13D & KR13+ & 6.236 & & & KR13+ & 5.615 & 5.926 \\
\hline РТ3284 & $R A S-W T$ & KR12+ & 1.553 & & & KR12+ & 1.606 & 1.5795 \\
\hline РТ3285 & KRASG12V & KR12+ & 23.79 & & & KR12+ & 19.804 & 21.797 \\
\hline \multicolumn{9}{|c|}{$\begin{array}{l}\text { Replicate aliquots of plasma samples that were centrally prepared were tested in either the GDL at the Christie Hospital (Manchester, UK), IMP laboratory (Kobe, Japan), or at the SI } \\
\text { Research and Development laboratory (Hamburg, Germany). } \\
\dagger \text { Dual replicates for certain patient samples were run at the IMP laboratory; these are indicated as two separate results to report both the qualitative RAS-WT or mRAS status for each } \\
\text { replicate, as well as the corresponding MAF values obtained in each replicate testing. Results obtained for patients } 3278 \text { and } 3279 \text {, indicated in boldface type are replicates that were } \\
\text { retested after carry-over contamination was noted in samples containing high MAF in adjacent samples; retesting confirmed that the RAS status for these two patients were indeed } \\
\text { RAS-WT. } \\
\ddagger \text { For patient } 3280 \text {, testing of the second replicate gave invalid results for certain codons; therefore, the overall result from this replicate was deemed invalid. } \\
\text { GDL: Genetics Diagnostics Laboratory; MAF: Mutant allelic fraction; RAS-WT: RAS wild-type; SI: Sysmex Inostics. }\end{array}$} \\
\hline
\end{tabular}

values in plasma were compared between newly diagnosed mCRC patients with intact primary tumor/metastases prior to surgery $(n=26)$ versus patients having either their primary tumors removed and/or experiencing recurrent disease, presenting with metastases $(n=22)$. A statistically significant relationship was observed between the patient clinical diagnosis status and mean proportion of mutant $R A S$ alleles in circulation. In stage IV newly diagnosed patients with intact primary tumors, the MAF was fivefold higher $(15.12 \%)$ than in patients presenting with recurrent disease after removal of their primary tumors $(3.03 \% ; \mathrm{p}=0.0001$; Figure 1$)$.

RAS mutational analysis in plasma in $\mathrm{mCRC}$ patients at progression

To examine changes in $R A S$ mutational status occurring in mCRC patients during treatment, ctDNA analyses were performed on blood samples obtained from patients with radiologically confirmed disease progression. Among 49 patients showing tumor tissue $R A S$ - WT status prior to chemotherapy, ctDNA analyses were performed on 24 patients (49\%) at disease progression. One sample was also taken from a patient who had a tissue NRAS mutation 
at baseline. 15 patients (30.6\%) having RAS-WT status died before a progression sample could be obtained and 10 patients (20.4\%) remained progression free and continued on first-line treatment. As shown in Table 6, a comparison of baseline plasma $R A S$ mutational status indicated that 23 out of $25 \mathrm{ctDNA}$ results were in agreement (92.0\% concordance; two patients showed a $R A S$ mutation in plasma but not in tissue). At progression, five out of these 25 patients (20\%) showed a change in mutational status in plasma as compared with the tissue and plasma $R A S$ test result obtained at baseline.

Plasma $R A S$ mutation testing revealed that 13 out of 16 patients receiving first-line anti-EGFR therapy $(81.2 \%)$ retained a $R A S-W T$ status at the time of progression and 3 of these patients (18.8\%) showed a change from $R A S-W T$ to mutated $R A S$ status at progression (Table 6). Two of the patients with RAS-WT status at baseline (2822 and 2817) received cetuximab + FOLFIRI and showed KRAS12 (MAF 2.5146) and KRAS13 mutations (MAF 0.3318), respectively, in plasma on progression. One patient (3274; Table 6) received Panitumumab + FOLFOX and showed a KRAS12 mutation in plasma (MAF $0.06 \%$ ) on progression, with an elevation in KRAS12 MAF to $0.4488 \%$ later during progression, along with evidence of two additional RAS mutations, KRAS61 (MAF 0.1031\%) and NRAS61 (MAF 0.356\%). These observations are consistent with the notion that failure of EGFR treatment in these patients likely resulted from expansion of multiple independent RAS-resistant clones at different metastatic sites. One patient with RAS-WT status at baseline treated with capecitabine showed a KRAS61 mutation in plasma at progression, indicating that a component of the resistance to treatment might involve $R A S$-mediated resistance.

Representative examples of the flow cytometry data from patients 2822 and 3274 showing quantitative readouts of $R A S$-mutation-positive versus wild-type molecular fractions detected by the OncoBEAM assay and emergence of $R A S$ mutations at progression are shown in Figure 2. These data show clearly the absence of RAS mutations in baseline samples obtained from both patients prior to anti-EGFR therapy treatments, as well as dramatic rises in $R A S$ mutant ctDNA molecules in plasma samples taken at progression.

\section{Discussion}

We have demonstrated that ctDNA RAS testing is a potential alternative to the current standard of mutation testing of FFPE material. This is supported by our results showing a high level of concordance (OPA 86\%) upon comparison of RAS mutation testing on prospectively collected plasma samples using the OncoBEAM RAS CRC assay versus those obtained using FFPE tumor samples. These results are in accord with findings from previous studies [12-14,19]. This is the first prospective study to examine both OncoBEAM RAS CEIVD assay performance in the $\mathrm{UK}$, as well as to monitor mCRC patients for changes in ctDNA $R A S$ mutational status at progression during first-line anti-EGFR therapy treatment.

The use of an extremely sensitive method to detect $R A S$ mutations in ctDNA from plasma provides several advantages over the standard tissue-based approach. Although primary tumor biopsy samples are routinely used for therapy selection, the use of such samples is subject to several technical and logistical limitations. For instance, a single-site tissue sample obtained at diagnosis may not fully represent disease heterogeneity and may not be informative of $R A S$ mutation status in those patients that relapse later with distant metastases. Intratumor heterogeneity is not insignificant: reports have demonstrated that testing DNA from a single colorectal tumor tissue block wrongly assigns $K R A S$ wild-type status in $8-11.6 \%$ of patients [20-22] Moreover, studies evaluating intertumor heterogeneity between primaries and metastases have also revealed mutational discordance in 3.6-32.4\% of cases [23-29]. Both inter- and intra-tumor heterogeneity are inherent features of metastatic disease [30-32] prudence would call fora systemic evaluation of RAS status across metastatic sites to better inform the accurate prescription of anti-EGFR therapy. Indeed, results support the utility of a liquid biopsy to overcome sampling bias associated with single tumor site-specific sampling [33].

In addition, locating appropriate tissue specimens for mutational testing at the time of therapy selection can present challenges and delay timely administration of a therapy, whereas the rapid turn-around time of plasma $R A S$ mutational testing enables timely initiation of first-line therapy.

There are obviously limitations in the use of ctDNA. First, the concentration of circulating-free DNA varies considerably between patients and the proportion of ctDNA within this is small $(0.1-10 \%)$ [34]. There is also a lack of standardization for ctDNA detection and analysis together with a lack of broadly accepted standard operating procedures [35]. In our study, analysis was duplicated in three different laboratories showing a high degree of consistency. Nonetheless, there are several clinical applications of liquid biopsies. These include genotyping for mutations, detecting minimal residual disease and helping to predict an early response to systemic chemotherapy for metastatic disease [34,36-38]. Furthermore, next-generation sequencing is able to rapidly and efficiently sequence 


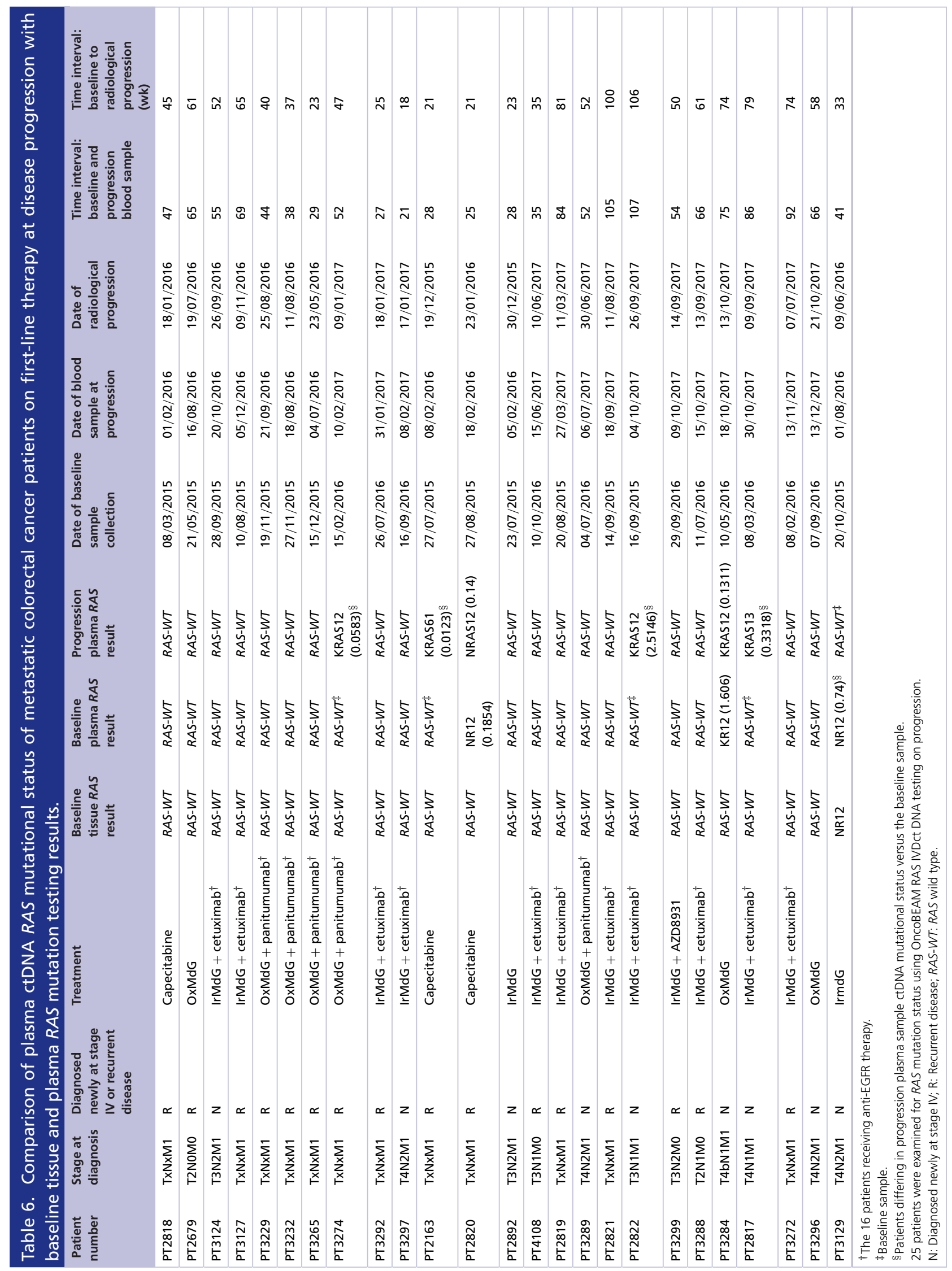



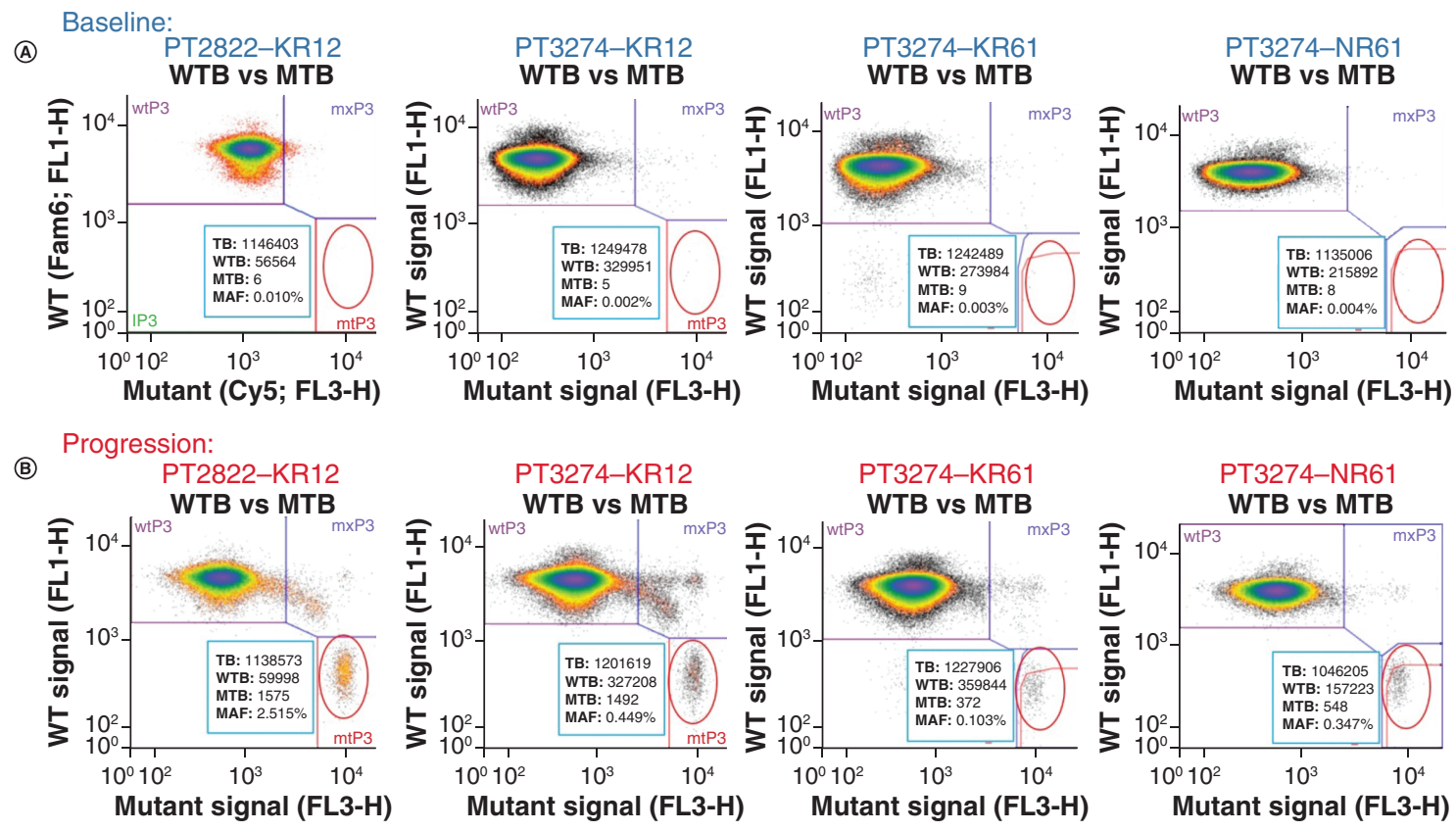

Figure 2. Quantitative measurement of RAS-mutation-positive versus wild-type ctDNA molecules in plasma samples obtained from patients 2822 and 3274 at baseline and disease progression. Comparison of RAS mutant (x-axis) versus WT (y-axis) flow cytometry analyses of ctDNA molecules detected on fluorescent beads using the OncoBEAM RAS IVD assay. Plots show the absolute numbers of TB, WTB, MTB and MAF percentages; these values from flow cytometric measurements are presented for each plot (see values in inset boxes within graphs). The eight plots presented are from data obtained in four different pairs (upper vs lower in a column) of ctDNA analyses of two different metastatic colorectal cancer patients (PT2822 and PT3274). PT2822 shows emergence of KRAS12 mutation at progression, whereas PT3274 shows emergence of three separate RAS mutations (KRAS12, KRAS61 and NRAS61) at progression. Plasma samples from these two patients measured at baseline are provided in panels shown in the top row denoted by (A), and samples measured at progression are provided in panels shown in the bottom row denoted by (B). Patterns of emerging RAS resistance of KR12, KR61 and NR61 mutations versus WT ctDNA in progression versus baseline measurements in these patients are visualized by comparing the patterns found in the lower with the upper plots, which also show ctDNA quantities in each of the four columns shown. Fluorescent signals from RAS mutant ctDNA molecules are observed as clustered signals in the lower right quadrants of each plot, where each cluster of ctDNA molecules is seen within the boundaries of the red ovals drawn in each plot. In contrast, the predominant pool of ctDNAs visualized and quantitated in each assay consist of WTBs; these are located in the upper left quadrants of each plot. For each assay, at least $1 \times 10^{6}$ beads are queried. Qualitative calls of WT (no mutation detected) or RAS mutation positive are determined by dividing the MTB counts (MB) by the WTB counts. The resulting ratio or MAF must exceed a cut-off value that has been derived for each of the codons detected in the assay. This concept is made clear with the data shown in the plots where the quantities of mutant molecules in baseline samples (plots in top row) are in the single digits (compare low MTB-MTB numbers to the number of WT molecules). Accordingly, the MAF values observed in baseline samples are below the cut-off thresholds set for each analyte. This pattern is in contrast to the RAS-positive calls made for the progression samples, where MTB counts are in the several hundred/thousands in comparison to similar levels of WT beads.

MAF: Mutant allelic fraction; MTB: Mutant beads; TB: Total beads; WTB: Wild-type beads.

millions of DNA reads using extremely low amounts of nucleic acids. This can lead to the identification of novel mutations or genomic rearrangements together with the possible evaluation of therapy response. Next-generation sequencing has also been used in the analysis of ctDNA for molecular profiling and tracking of minimal residual disease [39]. As part of the management of patients with CRC, it is important to know the mutational status of another gene, $B R A F$. In the EXPERT-C trial, 97 patients were assessable for the analysis of $K R A S / B R A F$ mutations in ctDNA. Only three patients were found to have $B R A F$ mutant tumors in the tissue samples, two of which were found to have mutations in the paired ctDNA samples [40].

Despite the significant therapeutic advances made by targeting EGFR pathways, most clinical benefit from anti-EGFR treatment is often short-lived due to the inevitable emergence of drug resistance. In RAS-WT mCRC patients receiving anti-EGFR therapy, the detection of $R A S$ mutations during therapy has been associated with 
disease progression [41-43]. In fact, using a liquid biopsy approach, independent research has demonstrated that $R A S$ mutant tumor DNA is detectable in plasma several months in advance of radiological progression $[11,41,43]$. Furthermore, mutated KRAS clones that emerge in blood during treatment with EGFR inhibitors decline on withdrawal of these antibodies [11,43]. Therefore, longitudinal ctDNA sampling for $R A S$ status can help to refine subsequent treatments. The ability to draw serial blood samples and perform ctDNA analyses from patients receiving anti-EGFR therapy provides signal opportunities to identify emerging $R A S$ mutant clones at early stages of treatment; this provides better visualization of treatment responses/failures that can further personalize therapy approaches for mCRC patients. In the present study, we showed that out of 25 progression samples taken, 5 (20\%) had a change in mutational status, with 3 out of 16 patients receiving anti-EGFR therapy showing RAS resistance to first-line treatment $(18.8 \%)$. These observations further underscore the importance of performing plasma testing in a longitudinal fashion during anti-EGFR therapy. Plasma RAS mutation monitoring would likely add benefit to the outcome/cost effectiveness of management of anti-EGFR treatment of CRC patients by providing a relatively inexpensive diagnostic tool to gauge therapy efficacy, switch therapy or schedule a timely treatment hiatus, particularly in patients who show early signs of $R A S$ resistance. The aggregated evidence therefore supports use of baseline and longitudinal ctDNA-based RAS mutation testing as an alternative to standard testing of FFPE material. This approach is likely to reduce unnecessary toxicity, cost and inconvenience, as well as to increase survival benefits for CRC patients receiving systemic therapy.

\title{
Conclusion
}

An accurate blood-based RAS mutation assay would benefit routine clinical management of mCRC patients to assist in guiding decisions to administer systemic therapy independent of the availability of tumor tissue samples. Here we used the OncoBEAM RAS IVD kit to show good concordance (86\%) of RAS status between tissue and plasma. We also showed that $20 \%$ of patients showed a change in their $R A S$ mutational status during treatment. These results demonstrate that blood-based $R A S$ mutation testing is a viable alternative to tissue-based $R A S$ testing and that there is a need for longitudinal plasma testing during treatment to guide management decisions and EGFR inhibitor administration.

\begin{abstract}
Author contributions
T Germetaki, M Braun, J Rogan, DL Edelstein, FS Jones and MP Saunders were responsible for trial design. T. Germetaki, C Nicholls, RA Adams, M Braun, J Rogan, S Moghadam and MP Saunders were responsible for sample acquisition. E Lenfert, A Lukas, DL Edelstein and FS Jones were responsible for sample analysis. T Germetaki, C Nicholls, RA Adams, M Braun, J Rogan, S Moghadam, E Lenfert, A Lukas, DL Edelstein, FS Jones and MP Saunders were responsible for data interpretation and manuscript drafting. Final approval was given by T Germetaki, C Nicholls, RA Adams, M Braun, J Rogan, S Moghadam, E Lenfert, A Lukas, DL Edelstein, FS Jones and MP Saunders. All agree to be accountable for all aspects of the work in ensuring that questions related to the accuracy or integrity of any part of the work are appropriately investigated and resolved.
\end{abstract}

\section{Summary points}

- Prior to initiation of anti-EGFR therapy, RAS (KRAS and NRAS) genotyping is performed and mutations are detected in approximately $50 \%$ of patients with metastatic colorectal cancer.

- Tissue processing presents several practical challenges, including poor tissue quality and from a biological perspective, accurate genotype determination can be obscured by tumor molecular heterogeneity.

- Performing ctDNA RAS testing is a potential alternative to the current standard of mutation testing of formalin-fixed paraffin embedded material.

- The OnCoBEAM ${ }^{\top M}$ RAS CRC plasma assay, based on the BEAMing technology [15], is liquid biopsy test that provides a systemic evaluation of RAS tumor mutation status from a blood sample in less than 7 days.

- The positive percentage agreement was $86.2 \%$ and the negative percentage agreement was $85.7 \%$.

- Our study showed a high overall percentage concordance $(86 \%)$ when comparing RAS mutation testing on prospectively collected plasma samples using the OncoBEAM RAS CRC assay with formalin-fixed paraffin embedded tumor samples.

- Reproducibility studies showed that OncoBEAM testing results had $100 \%$ agreement between three independent laboratories.

- $20 \%$ of patients showed changes in their RAS mutational status during treatment.

- Overall, these results show good concordance between tissue and plasma samples and suggest the need for longitudinal plasma testing during treatment to guide management decisions during anti-EGFR therapy. 


\section{Acknowledgments}

The authors would like to acknowledge the Manchester Cancer Research Biobank for collecting and storing samples. Research samples were obtained from the Manchester Cancer Research Centre (MCRC) Biobank, UK. The role of the MCRC Biobank is to distribute samples and therefore cannot endorse studies performed or the interpretation of results. We would also like to acknowledge the patients who consented to be part of this study.

\section{Financial \& competing interests disclosure}

This study was cooperatively funded by Sysmex Inostics and Merck KGaA. They provided money to allow collection of the samples for testing and analysis of the blood samples. Sysmex Inc. funded the Open Access License fees for the current journal. T Germetaki received honoraria from Merck and Servier, MP Saunders received honoraria from Merck, Amgen and Servier. RA Adams received consultancy fees from Merck Serono, AstraZeneca, Amgen and Servier. FS Jones is an employee of Sysmex America, Inc. DL Edelstein, E Lenfert and A Lukas are employees of Sysmex Inostics. The authors have no other relevant affiliations or financial involvement with any organization or entity with a financial interest in or financial conflict with the subject matter or materials discussed in the manuscript apart from those disclosed.

No writing assistance was utilized in the production of this article.

\section{Ethical conduct of research}

The authors state that they have obtained appropriate approval from Manchester Cancer Research Biobank. For investigations involving human subjects, informed consent has been obtained from the participants involved.

\section{Data sharing statement}

This manuscript presents original results from sample analysis collected via the MCRC as described above in the acknowledgments.

\section{Open access}

This work is licensed under the Attribution-NonCommercial-NoDerivatives 4.0 Unported License. To view a copy of this license, visit http://creativecommons.org/licenses/by-nc-nd/4.0/

\section{References}

Papers of special note have been highlighted as: $\bullet$ of interest; $\bullet \bullet$ of considerable interest

1. Cunningham D, Humblet $Y$, Siena $S$ et al. Cetuximab monotherapy and cetuximab plus irinotecan in irinotecan-refractory metastatic colorectal cancer. N. Engl. J. Med. 351(4), 337-345 (2004).

2. Saltz LB, Meropol NJ, Loehrer PJ, Needle MN, Kopit J, Mayer RJ. Phase II trial of cetuximab in patients with refractory colorectal cancer that expresses the epidermal growth factor receptor. J. Clin. Oncol. 22(7), 1201-1208 (2004).

3. Tabernero J, van Cutsem E, Díaz-Rubio E et al. Phase II trial of cetuximab in combination with fluorouracil, leucovorin and oxaliplatin in the first-line treatment of metastatic colorectal cancer. J. Clin. Oncol. 25(33), 5225-5232 (2007).

4. Kafatos G, Niepel D, Lowe K et al. RAS mutation prevalence among patients with metastatic colorectal cancer: a meta-analysis of real-world data. Biomark. Med. 11(9), 751-760 (2017).

- Introduces the $R A S$ mutation prevalence among patients with metastatic colorectal cancer.

5. Peeters M, Kafatos G, Taylor A et al. Prevalence of RAS mutations and individual variation patterns among patients with metastatic colorectal cancer: a pooled analysis of randomised controlled trials. Eur. J. Cancer 51(13), 1704-1713 (2015).

6. Malapelle U, Carlomagno C, de Luca C, Bellevicine C, Troncone G. KRAS testing in metastatic colorectal carcinoma: challenges, controversies, breakthroughs and beyond. J. Clin. Pathol. 67(1), 1-9 (2013).

7. Tack V, Ligtenberg MJL, Tembuyser L et al. External quality assessment unravels interlaboratory differences in quality of RAS testing for anti-EGFR therapy in colorectal cancer. Oncologist 20(3), 257-262 (2015).

8. Gerlinger M, Rowan AJ, Horswell S et al. Intratumor heterogeneity and branched evolution revealed by multiregion sequencing. $N$. Engl. J. Med. 366(10), 883-892 (2012).

-. Highlights the role of intratumor heterogeneity in personalized medicine approaches

9. Wang S, An T, Wang J et al. Potential clinical significance of a plasma-based KRAS mutation analysis in patients with advanced non-small cell lung cancer. Clin. Cancer Res. 16(4), 1324-1330 (2010).

10. Bettegowda C, Sausen M, Leary RJ et al. Detection of circulating tumor DNA in early- and late-stage human malignancies. Sci. Trans. Med. 6(224), 224ra24 (2014).

11. Siravegna G, Mussolin B, Buscarino $M$ et al. Clonal evolution and resistance to EGFR blockade in the blood of colorectal cancer patients. Nat. Med. 217), 795-801 2015). 
12. García-Foncillas J, Tabernero J, Élez E et al. Prospective multicenter real-world RAS mutation comparison between OncoBEAM-based liquid biopsy and tissue analysis in metastatic colorectal cancer. Br. J. Cancer 119, 1464-1470 (2018).

13. Schmiegel W, Scott RJ, Dooley $S$ et al. Blood-based detection of RAS mutations to guide anti-EGFR therapy in colorectal cancer patients: concordance of results from circulating tumor DNA and tissue-based RAS testing. Mol. Oncol. 11(2), 208-219 (2017).

14. Vidal J, Muinelo L, Dalmases A et al. Plasma ctDNA RAS mutation analysis for the diagnosis and treatment monitoring of metastatic colorectal cancer patients. Ann. Oncol. 28(6), 1325-1332 (2017).

15. Diehl F, Li M, He Y, Kinzler KW, Vogelstein B, Dressman D. BEAMing: single-molecule PCR on microparticles in water-in-oil emulsions. Nat. Methods 3(7), 551-559 (2006).

16. van Cutsem E, Lenz H-J, Köhne C-H et al. Fluorouracil, leucovorin and irinotecan plus cetuximab treatment and RAS mutations in colorectal cancer. J. Clin. Oncol. 33(7), 692-700 (2015).

-. The research progress of metastatic colorectal cancer was introduced.

17. Bokemeyer C, Kohne C-H, Ciardiello F et al. Treatment outcome according to tumor RAS mutation status in OPUS study patients with metastatic colorectal cancer (mCRC) randomized to FOLFOX4 with/without cetuximab. J. Clin. Oncol. 32(Suppl. 15), 3505-3505 (2014).

18. Dressman D, Yan H, Traverso G, Kinzler KW, Vogelstein B. Transforming single DNA molecules into fluorescent magnetic particles for detection and enumeration of genetic variations. Proc. Natl Acad. Sci. USA 100(15), 8817-8822 (2003).

19. Bando $\mathrm{H}$, Kagawa $\mathrm{Y}$, Kato $\mathrm{T}$ et al. A multicentre, prospective study of plasma circulating tumour DNA test for detecting RAS mutation in patients with metastatic colorectal cancer. Br. J. Cancer 120(10), 982-986 (2019).

20. Baldus SE, Schaefer KL, Engers R, Hartleb D, Stoecklein NH, Gabbert HE. Prevalence and heterogeneity of KRAS, BRAF and PIK3CA mutations in primary colorectal adenocarcinomas and their corresponding metastases. Clin. Cancer Res. 16(3), 790-799 (2010).

21. Richman SD, Chambers P, Seymour MT et al. Intra-tumoral heterogeneity of KRAS and BRAF mutation status in patients with advanced colorectal cancer (aCRC) and cost-effectiveness of multiple sample testing. Anal. Cell. Pathol. 34(1-2), 61-66 (2011).

22. Watanabe T, Kobunai T, Yamamoto Y, Matsuda K et al. Heterogeneity of KRAS status may explain the subset of discordant KRAS status between primary and metastatic colorectal cancer. Dis. Colon Rectum 54(9), 1170-1178 (2011).

23. Lamy A, Blanchard F, le Pessot F et al. Metastatic colorectal cancer KRAS genotyping in routine practice: results and pitfalls. Mod. Pathol. 24(8), 1090-1100 (2011).

24. Knijn N, Mekenkamp LJM, Klomp M et al. KRAS mutation analysis: a comparison between primary tumours and matched liver metastases in 305 colorectal cancer patients. Br. J. Cancer 104(6), 1020-1026 (2011).

25. Artale S, Sartore-Bianchi A, Veronese SM et al. Mutations of KRAS and BRAF in primary and matched metastatic sites of colorectal cancer. J. Clin. Oncol. 26(25), 4217-4219 (2008).

26. Mostert B, Jiang Y, Sieuwerts AM et al. KRAS and BRAF mutation status in circulating colorectal tumor cells and their correlation with primary and metastatic tumor tissue. Int. J. Cancer 133(1), 130-141 (2013).

27. Italiano A, Hostein I, Soubeyran I et al. KRAS and BRAF mutational status in primary colorectal tumors and related metastatic sites: biological and clinical implications. Ann. Surg. Oncol. 17(5), 1429-1434 (2010).

28. Kim MJ, Lee HS, Kim JH et al. Different metastatic pattern according to the KRAS mutational status and site-specific discordance of KRAS status in patients with colorectal cancer. BMC Cancer 12, 347 2012).

29. Tie J, Lipton L, Desai J et al. KRAS mutation is associated with lung metastasis in patients with curatively resected colorectal cancer. Clin. Cancer Res. 17(5), 1122-1130 (2011).

30. Gerlinger M, Catto JW, Orntoft TF, Real FX, Zwarthoff EC, Swanton C. Intratumour heterogeneity in urologic cancers: from molecular evidence to clinical implications. Eur. Urol. 67(4), 729-737 (2015).

31. de Mattos-Arruda L, Weigelt B, Cortes J et al. Capturing intra-tumor genetic heterogeneity by de novo mutation profiling of circulating cell-free tumor DNA: a proof-of-principle. Ann. Oncol. 25(9), 1729-1735 (2014).

32. Vogelstein B, Papadopoulos N, Velculescu VE, Zhou S, Diaz LA, Kinzler KW. Cancer genome landscapes. Science 339(6127), 1546-1558 (2013).

33. Russo M, Siravegna G, Blaszkowsky LS et al. Tumor heterogeneity and lesion-specific response to targeted therapy in colorectal cancer. Cancer Discov. 6(2), 147-153 (2016).

34. Diehl F, Schmidt K, Choti MA et al. Circulating mutant DNA to assess tumor dynamics. Nat. Med. 14(9), 985-990 (2008).

35. Crowley E, di Nicolantonio F, Loupakis F, Bardelli A. Liquid biopsy: monitoring cancer-genetics in the blood. Nat. Rev. Clin Oncol. 10(8), 472-484 (2013).

36. Reckamp KL, Melnikova VO, Karlovich C et al. A highly sensitive and quantitative test platform for detection of NSCLC EGFR mutations in urine and plasma. J. Thorac. Oncol. 11(10), 1690-1700 (2016).

37. Tie J, Kinde I, Wang Y et al. Circulating tumor DNA as an early marker of therapeutic response in patients with metastatic colorectal cancer. Ann. Oncol. 26(8), 1715-1722 (2015). 
38. Siravegna G, Mussolin B, Venesio T et al. How liquid biopsies can change clinical practice in oncology. Ann. Oncol. 30(10), 1580-1590 (2019).

- Liquid biopsies and their role in understanding the tumor evolution.

39. Tarazona N, Gimeno-Valiente F, Gambardella V et al. Targeted next-generation sequencing of circulating-tumor DNA for tracking minimal residual disease in localized colon cancer. Ann. Oncol. 30(11), 1804-1812 (2019).

40. Sclafani F, Chau I, Cunningham D et al. KRAS and BRAF mutations in circulating tumour DNA from locally advanced rectal cancer. Sci.Rep. 8(1), 1445 (2018).

41. Diaz LA, Williams RT, Wu J et al. The molecular evolution of acquired resistance to targeted EGFR blockade in colorectal cancers. Nature 486(7404), 537-540 (2012).

-. Diaz et al. described the development of acquired resistance to epidermal growth factor receptor blockade as a result of the emergence of KRAS mutations.

42. Misale $S$, Yaeger R, Hobor $S$ et al. Emergence of KRAS mutations and acquired resistance to anti-EGFR therapy in colorectal cancer. Nature 486(7404), 532-536 (2012).

43. Morelli MP, Overman MJ, Dasari A et al. Characterizing the patterns of clonal selection in circulating tumor DNA from patients with colorectal cancer refractory to anti-EGFR treatment. Ann. Oncol. 26(4), 731-736 (2015).

-• Morelli et al. described the correlation of shorter progression-free survival with epidermal growth factor receptor blockade treatment in patients with low-frequency KRAS mutation expression in archival tissue and plasma samples. 
